\title{
BERS ISOMORPHISM ON THE UNIVERSAL TEICHMÜLLER CURVE
}

\author{
LEE-PENG TEO
}

\begin{abstract}
We study the Bers isomorphism between the Teichmüller space of the parabolic cyclic group and the universal Teichmüller curve. We prove that this is a group isomorphism and its derivative map gives a remarkable relation between Fourier coefficients of cusp forms and Fourier coefficients of vector fields on the unit circle. We generalize the Takhtajan-Zograf metric to the Teichmüller space of the parabolic cyclic group, and prove that up to a constant, it coincides with the pull back of the Velling-Kirillov metric defined on the universal Teichmüller curve via the Bers isomorphism.
\end{abstract}

\section{INTRODUCTION}

Let $\mathbb{U}$ be the upper half plane and $G$ a Fuchsian group. We denote by $T(G)$ the Teichmüller space of $G$ and $F(G)$ the Bers fiber space of $G$. In Ber73, Bers proved the following result.

Theorem 1.1. Let $G$ be a torsion free Fuchsian group, a a point in $\mathbb{U}, \hat{a}$ the image of a under the natural projection $\mathbb{U} \rightarrow \mathbb{U} / G$. Let $\dot{G}$ be another torsion free Fuchsian group and $h: \mathbb{U} / \dot{G} \rightarrow(\mathbb{U} / G) \backslash\{\hat{a}\}$ a conformal bijection. Then there is a canonical biholomorphic bijection $T(\dot{G}) \rightarrow F(G)$.

In case $G=\{\mathrm{id}\}$ is the trivial group, Bers theorem implies a biholomorphic bijection $T\left(\Gamma_{0}\right) \rightarrow F(1)$, where $\Gamma_{0}$ is the parabolic subgroup $\{z \mapsto$ $z+n \mid n \in \mathbb{Z}\}$ of $\operatorname{PSL}(2, \mathbb{R})$, and $F(1)$ is the universal Teichmüller curve. Both the universal Teichmüller space $T(1)$ and the universal Teichmüller curve $F(1)$ have group structures. We are going to show in Section 2 that as a subspace of $T(1), T\left(\Gamma_{0}\right)$ is also a subgroup. Moreover, we prove in Section 3 that the Bers isomorphism on $T\left(\Gamma_{0}\right)$ is a group isomorphism.

In Teo04, we proved that the universal Teichmüller curve $F(1)$ is isomorphic to the homogenuous space $S^{1} \backslash \operatorname{Homeo}_{q s}\left(S^{1}\right)$, where $\operatorname{Homeo}_{q s}\left(S^{1}\right)$ is the space of quasi-symmetric homeomorphisms of the unit circle. Using this isomorphism, we prove in Section 4 that the derivative of the Bers isomorphism at the origin of $T\left(\Gamma_{0}\right)$ gives a remarkable relation between the Fourier

Date: December 19, 2017.

2000 Mathematics Subject Classification. Primary 30F60; Secondary 32G15.

Key words and phrases. universal Teichmüller curve, Teichmüller space, Bers isomorphism, Velling-Kirillov metric, Takhtajan-Zograf metric. 
coefficients of cusp forms on the upper half plane and Fourier coefficients of vector fields on the unit circle.

In Teo04, we defined the Velling-Kirillov metric on the universal Teichmüller curve $F(1)$, which we proved is the unique right invariant Kähler metric on $F(1)$. In [TZ91, Takhtajan and Zograf defined a new Kähler metric on $T_{g, n}$, the Teichmüller space of Riemann surfaces of genus $g$ with $n$ punctures, which plays an important role in the local index theorem. In Section 2, we generalize the definition of this metric to $T\left(\Gamma_{0}\right)$ and still call it the Takhtajan-Zograf metric. By definition, this metric is right-invariant. Hence it is not surprising that we have the following theorem (see Theorem 5.1).

Theorem 1.2. The pull back of the Velling-Kirillov metric on $F(1)$ via the biholomorphic bijection $T\left(\Gamma_{0}\right) \rightarrow F(1)$ is a multiple of the Takhtajan-Zograf metric on $T\left(\Gamma_{0}\right)$.

\section{TeICHMÜlleR SPACES AND KÄHLER METRICS}

In this section, we review the results we need from Teichmüller theory and our previous paper Teo04. The readers can consult standard texts in Teichmüller theory Leh87, Nag88 or our papers Teo04, TT03, for further details.

Let $\mathbb{U}, \mathbb{L}, \mathbb{D}$ and $\mathbb{D}^{*}$ be respectively the upper half plane, the lower half plane, the unit disc and the exterior of unit disc. In this paper, we are going to use both the upper half plane model and the unit disc model for the universal Teichmüller space $T(1)$. In case of the disc model, it is described as follows. Let $L^{\infty}(\mathbb{D})$ be the complex Banach space of bounded Beltrami differentials on $\mathbb{D}$. We denote by $L^{\infty}(\mathbb{D})_{1}$ the unit ball of $L^{\infty}(\mathbb{D})$. For $\mu \in L^{\infty}(\mathbb{D})_{1}$, we extend $\mu$ to $\mathbb{D}^{*}$ by reflection

$$
\mu(z)=\overline{\mu\left(\frac{1}{\bar{z}}\right)} \frac{z^{2}}{\bar{z}^{2}}, \quad z \in \mathbb{D}^{*} .
$$

There is a unique quasi-conformal mapping $w_{\mu}$ fixing $-1,-i, 1$ which solves the Beltrami equation

$$
\left(w_{\mu}\right)_{\bar{z}}=\mu\left(w_{\mu}\right)_{z} .
$$

It fixes the unit circle $S^{1}, \mathbb{D}$ and $\mathbb{D}^{*}$. The universal Teichmüller space is defined as a set of equivalence classes of normalized quasi-conformal mappings

$$
T(1)=L^{\infty}(\mathbb{D})_{1} / \sim
$$

where $\mu \sim \nu$ if and only if $w_{\mu}=w_{\nu}$ on the unit circle. The tangent space at identity is identified with $\Omega^{-1,1}(\mathbb{D})$, the space of harmonic Beltrami differentials on $\mathbb{D}$. More explicitly, let

$$
A_{\infty}(\mathbb{D})=\left\{\phi \text { holomorphic on } \mathbb{D}\left|\sup _{z \in \mathbb{D}}\right|\left(1-|z|^{2}\right)^{2} \phi(z) \mid<\infty\right\}
$$


be the space of bounded holomorphic quadratic differentials on $\mathbb{D}$. Then

$$
\Omega^{-1,1}(\mathbb{D})=\left\{-\frac{\left(1-|z|^{2}\right)^{2}}{2} \overline{\phi(z)} \mid \phi \in A_{\infty}(\mathbb{D})\right\} .
$$

The upper half plane model for the Teichmüller space $T(1) \simeq L^{\infty}(\mathbb{U})_{1} / \sim$ is defined analogously where we replace $\mathbb{D}$ and $\mathbb{D}^{*}$ in the definition above by $\mathbb{U}$ and $\mathbb{L}$ respectively; the reflection on $\mu$ is defined by $\mu(z)=\overline{\mu(\bar{z})}$; the quasi-conformal mapping $w_{\mu}$ is normalized to fix the points $0,1, \infty$ and two quasi-conformal mappings $w_{\mu}$ and $w_{\nu}$ are equivalent if and only if they agree on the extended real line $\hat{\mathbb{R}}=\mathbb{R} \cup\{\infty\}$.

The universal Teichmüller curve $F(1)$ is defined as

$$
F(1)=\left\{([\mu], z) \mid[\mu] \in T(1), z \in f^{\mu}(\mathbb{D})\right\} .
$$

Here $f^{\mu}$ is the quasiconformal map that is holomorphic on $\mathbb{D}^{*}$, normalized such that $f(\infty)=\infty, f^{\prime}(\infty)=1,(f-z)(\infty)=0$ and satisfies the Beltrami equation $f_{\bar{z}}=\mu f_{z}$ on $\mathbb{D}$. The tangent space at identity is identified with $\Omega^{-1,1}(\mathbb{D}) \oplus \mathbb{C}$.

Let Homeo $_{q s}\left(S^{1}\right)$ be the space of quasi-symmetric homeomorphisms on the unit circle, i.e. it consists of orientation preserving homeomorphism of the unit circle $\omega: S^{1} \rightarrow S^{1}$ that satisfies

$$
\frac{1}{M} \leq \frac{\omega\left(e^{2 \pi i(x+t)}\right)-\omega\left(e^{2 \pi i x}\right)}{\omega\left(e^{2 \pi i x}\right)-\omega\left(e^{2 \pi i(x-t)}\right)} \leq M, \quad \forall x, t \in \mathbb{R}, 0<t<\frac{1}{4},
$$

for some constant $M$. We identify $S^{1} \backslash \operatorname{Homeo}_{q s}\left(S^{1}\right)$ as the subspace of Homeo $_{q s}\left(S^{1}\right)$ consisting of those $\omega$ that fix the point 1 . Every $\omega \in S^{1} \backslash \operatorname{Homeo}_{q s}\left(S^{1}\right)$ can be extended to be a symmetric (with respect to $S^{1}$ ) quasi-conformal mapping which we still denote by $\omega$. It has a unique conformal welding $\omega=g^{-1} \circ f$ satisfying the conditions 1) $f$ and $g$ are quasi-conformal mappings. 2) $\left.f\right|_{\mathbb{D}^{*}}$ and $\left.g\right|_{\mathbb{D}}$ are conformal and depend only on $\left.\omega\right|_{S^{1}}$. 3) $f(\infty)=\infty, f^{\prime}(\infty)=1, g(0)=0$. We call $\left(\left.f\right|_{\mathbb{D}^{*}},\left.g\right|_{\mathbb{D}}\right)$ the pair of univalent functions associated to the point $\omega \in S^{1} \backslash \mathrm{Homeo}_{q s}\left(S^{1}\right)$, which we also write as $(f, g)$. We identify $T(1)$ as the subspace of $S^{1} \backslash \operatorname{Homeo}_{q s}\left(S^{1}\right)$ consisting of those $\omega$ whose corresponding $f$ satisfies the additional condition $(f-z)(\infty)(0)$, i.e. the power series expansion of $f$ has the form

$$
f(z)=z+\frac{b_{1}}{z}+\frac{b_{2}}{z^{2}}+\ldots, \quad z \in \mathbb{D}^{*} .
$$

In fact, for every $\mu \in L^{\infty}(\mathbb{D})_{1}$ extended by symmetry to $\mathbb{D}^{*}$, there exists a unique symmetric quasi-conformal mapping $\omega$ that satisfies the Beltrami equation $\omega_{\bar{z}}=\mu \omega_{z}$ and whose corresponding $f$ in the conformal welding of $\omega$ has the form (2.2). We denote this $\omega$ by $\omega_{\mu}$ and the corresponding conformal welding by $\left(f^{\mu}, g_{\mu}\right)$. The isomorphism $\mathfrak{T}: F(1) \stackrel{\sim}{\rightarrow} S^{1} \backslash \operatorname{Homeo}_{q s}\left(S^{1}\right)$ is given by

$$
\left.F(1) \ni([\mu], z) \mapsto \sigma_{w} \circ \omega_{\mu}\right|_{S^{1}} \in S^{1} \backslash \operatorname{Homeo}_{q s}\left(S^{1}\right),
$$


where $w=g_{\mu}^{-1}(z) \in \mathbb{D}$ and

$$
\sigma_{w}(\zeta)=\frac{1-\bar{w}}{1-w} \frac{\zeta-w}{1-\zeta \bar{w}} \in \operatorname{PSU}(1,1) .
$$

For the purpose of next section, we introduce the space

$$
\mathcal{Q}=\left\{\omega_{\mu} \mid \mu \in L^{\infty}(\mathbb{D})_{1}\right\}
$$

From our reasoning above, it is isomorphic to $L^{\infty}(\mathbb{D})_{1}$ via the canonical correspondence $\mu \mapsto \omega_{\mu}$. Moreover, there is a real analytic isomorphism $\mathfrak{J}: \mathcal{Q} / \sim \times \mathbb{D} \rightarrow F(1)$ given by

$$
\mathcal{Q} / \sim \times \mathbb{D} \ni\left(\left[\omega_{\mu}\right], z\right) \mapsto\left([\mu], g_{\mu}(z)\right) \in F(1),
$$

where the equivalence relation $\sim$ on $\mathcal{Q}$ is defined in the obvious way: $\omega_{\mu} \sim$ $\omega_{\nu}$ if and only if $\omega_{\mu}$ and $\omega_{\nu}$ agree on $S^{1}$. We also define $\mathfrak{K}: \mathcal{Q} \times \mathbb{D} \rightarrow$ $S^{1} \backslash \operatorname{Homeo}_{q s}\left(S^{1}\right)$ by $\left.\left(\omega_{\mu}, z\right) \mapsto \sigma_{z} \circ \omega_{\mu}\right|_{S^{1}}$. Obviously, $\mathfrak{K}=\mathfrak{T} \circ \mathfrak{J} \circ \Pi$, where $\Pi: \mathcal{Q} \times \mathbb{D} \rightarrow \mathcal{Q} / \sim \times \mathbb{D}$ is the canonical projection.

The tangent space at identity of $S^{1} \backslash \operatorname{Homeo}_{q s}\left(S^{1}\right)$ is identified with the Zygmund class vector fields ${ }^{1}$ on $S^{1}$. Given a one parameter flow $\omega_{t} \in$ $S^{1} \backslash \operatorname{Homeo}_{q s}\left(S^{1}\right)$ where $\omega_{0}=\mathrm{id}$, it defines the tangent vector

$$
v=u\left(e^{i \theta}\right) \frac{\partial}{\partial \theta}=\sum_{n \in \mathbb{Z}} c_{n} e^{i n \theta} \frac{\partial}{\partial \theta}
$$

where $u$ is defined by $\dot{\omega}(z)=\left.\frac{d}{d t}\right|_{t=0} \omega_{t}(z)=i z u(z)$. Since $\omega_{t}\left(S^{1}\right)=S^{1}$ and $\omega_{t}(1)=1$ for all $t$, we find that

$$
c_{-n}=\bar{c}_{n} \quad \text { and } \quad \sum_{n \in \mathbb{Z}} c_{n}=0 .
$$

The Velling-Kirillov metric is a right invariant metric on $S^{1} \backslash \operatorname{Homeo}_{q s}\left(S^{1}\right)$. At the origin, it is given by

$$
\|v\|_{V K}^{2}=\sum_{n=1}^{\infty} n\left|c_{n}\right|^{2} .
$$

The group structure on $T(1) \simeq L^{\infty}(\mathbb{U})_{1} / \sim$ is induced by the composition of quasi-conformal mappings, i.e. $[\mu] *[\nu]=[\lambda]$ if and only if $w_{\mu} \circ w_{\nu} \sim w_{\lambda}$; whereas the group structure on $F(1)$ is induced from the group structure on $S^{1} \backslash \operatorname{Homeo}_{q s}\left(S^{1}\right)$, which is defined by composition of quasi-symmetric homeomorphisms.

Let $\Gamma_{0}$ be the parabolic cyclic group $\Gamma_{0}=\{z \mapsto z+n \mid n \in \mathbb{Z}\}$. The Teichmüller space $T\left(\Gamma_{0}\right)$ is defined as

$$
L^{\infty}\left(\mathbb{U}, \Gamma_{0}\right)_{1} / \sim,
$$

where $L^{\infty}\left(\mathbb{U}, \Gamma_{0}\right)_{1}$ is the subspace of $L^{\infty}(\mathbb{U})_{1}$ consisting of those $\mu$ satisfying

$$
\mu(z+1)=\mu(z), \quad z \in \mathbb{U}
$$

\footnotetext{
${ }^{1}$ See [Teo04] for definition.
} 
and $\sim$ is the same equivalence relation defined on $L^{\infty}(\mathbb{U})_{1}$.

Given $[\mu] \in T\left(\Gamma_{0}\right)$, let $\Gamma_{\mu}=w_{\mu} \circ \Gamma_{0} \circ w_{\mu}^{-1}$. It is a parabolic cyclic subgroup of $\operatorname{PSL}(2, \mathbb{R})$ generated by

$$
\gamma_{0}=w_{\mu} \circ \theta_{0} \circ w_{\mu}^{-1}, \quad \theta_{0}(z)=z+1 .
$$

Putting $z=0$ and $z \rightarrow \infty$ in the relation $\gamma_{0} \circ w_{\mu}=w_{\mu} \circ \theta_{0}$, we find that $\gamma_{0}(0)=1$ and $\gamma_{0}(\infty)=\infty$. Consequently, $\gamma_{0}=\theta_{0}, \Gamma_{\mu}=\Gamma_{0}$ and $w_{\mu}(z+1)=w_{\mu}(z)+1$. As a result, $T\left(\Gamma_{0}\right)$ can be equivalently defined as equivalence classes of symmetric quasi-conformal mappings $w_{\mu}$ satisfying the condition $w_{\mu}(z+1)=w_{\mu}(z)+1$. Then it is easily seen that $T\left(\Gamma_{0}\right)$ is a subgroup of $T(1)$.

Remark 2.1. It is well known that the universal Teichmüller space $T(1)$ is isomorphic to the space of quasi-symmetric homeomorphisms on $\hat{\mathbb{R}}$ fixing $0,1, \infty$, where a homeomorphism $u: \mathbb{R} \rightarrow \mathbb{R}$ is called quasi-symmetric if and only if $u$ is monotonically increasing and there exists a constant $M$ such that

$$
\frac{1}{M} \leq \frac{u(x+h)-u(x)}{u(x)-u(x-h)} \leq M \quad \forall x, h \in \mathbb{R}, h>0 .
$$

The isomorphism is given by $\left.[\mu] \mapsto w_{\mu}\right|_{\mathbb{R}}$. The subspace $T\left(\Gamma_{0}\right)$ is then identified with the space of quasi-symmetric homeomorphisms on $\mathbb{R}$ that fix all the integers and satisfy the condition $u(x+1)=u(x)+1$ for all $x$.

The tangent space at the origin of $T\left(\Gamma_{0}\right)$ is identified with

$$
\Omega^{-1,1}\left(\mathbb{U}, \Gamma_{0}\right)=\left\{-2 y^{2} \bar{\phi}: \phi \in A_{\infty}\left(\mathbb{U}, \Gamma_{0}\right)\right\},
$$

where $A_{\infty}\left(\mathbb{U}, \Gamma_{0}\right)$ is the space of cusp forms

$$
A_{\infty}\left(\mathbb{U}, \Gamma_{0}\right)=\left\{\phi: \mathbb{U} \rightarrow \mathbb{C} \text { holomorphic }\left|\sup _{z \in \mathbb{U}}\right| y^{2} \phi(z) \mid<\infty, \phi(z+1)=\phi(z)\right\} \text {. }
$$

In [TZ91, Takhtajan and Zograf defined a new Kähler metric on $T_{g, n}$, the Teichmüller space of Riemann surfaces of genus $g$ with $n$ punctures. We generalize this metric to be a right invariant metric on $T\left(\Gamma_{0}\right)$. At the origin, it is defined by

$$
\langle\mu, \nu\rangle_{T Z}=\iint_{F} \mu(z) \overline{\nu(z)} d^{2} z, \quad \mu, \nu \in \Omega^{-1,1}\left(\mathbb{U}, \Gamma_{0}\right) .
$$

Here $F=\{x+i y \mid 0<x<1,0<y<\infty\}$ is a fundamental domain of $\Gamma_{0}$ on $\mathbb{U}$. By definition, $\mu=-2 y^{2} \bar{\phi}$, where $\phi \in A_{\infty}\left(\mathbb{U}, \Gamma_{0}\right)$ and therefore has an expansion

$$
\phi(z)=\sum_{n=1}^{\infty} \alpha_{n} e^{2 \pi i n z}
$$

A direct computation gives

$$
\|\mu\|_{T Z}^{2}=\frac{3}{32 \pi^{5}} \sum_{n=1}^{\infty} \frac{\left|\alpha_{n}\right|^{2}}{n^{5}}
$$




\section{Bers Isomorphism Between $T\left(\Gamma_{0}\right)$ And $F(1)$.}

In this section, we are going to give a separate proof of the Bers isomorphism $\mathfrak{B}: T\left(\Gamma_{0}\right) \rightarrow F(1)$. The basic idea follows the same line as in [Ber73]. But we use a different model for $F(1)$. Besides, the proof is much simpler in this case. On the other hand, we are also going to show that $\mathfrak{B}$ is in fact a group isomorphism.

Let $p(z)=e^{2 \pi i z}$. Then $p: \mathbb{U} \rightarrow \mathbb{D} \backslash\{0\}$ is a holomorphic covering map which can be extended to be a continuous map $p: \mathbb{U} \cup \mathbb{R} \rightarrow \mathbb{D} \cup S^{1} \backslash\{0\}$. Given $\mu \in L^{\infty}\left(\mathbb{U}, \Gamma_{0}\right)_{1}$, let $\nu$ be defined such that

$$
\nu \circ p \frac{\overline{p^{\prime}}}{p^{\prime}}=\mu \text {. }
$$

This is well defined in view of the periodicity of $\mu$. We define the pre-Bers mapping $B: L^{\infty}\left(\mathbb{U}, \Gamma_{0}\right)_{1} \rightarrow \mathcal{Q} \times \mathbb{D}$ by $\mu \rightarrow\left(\omega_{\nu}, \omega_{\nu}(0)\right)$. Then there exists a unique holomorphic covering map $p_{\mu}: \mathbb{U} \rightarrow \mathbb{D} \backslash\left\{w_{\nu}(0)\right\}$ such that the following diagram

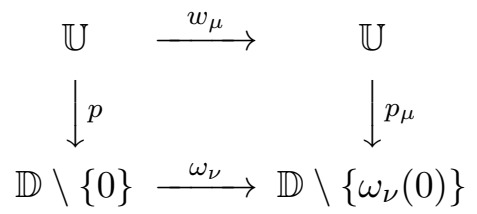

is commutative, i.e.

$$
p_{\mu} \circ w_{\mu}(z)=w_{\nu} \circ p(z), \quad z \in \mathbb{U} .
$$

This equation can be extended continuously to $\mathbb{U} \cup \mathbb{R}$. By uniqueness of holomorphic mappings, we have $p_{\mu}=\gamma_{\mu} \circ p$, where $\gamma_{\mu} \in \operatorname{PSU}(1,1)$ is a fractional linear transformation that maps 0 to $\omega_{\nu}(0)$. Moreover, putting $z=0$ in (3.3), we find that $\gamma_{\mu}(1)=1$. This determine $\gamma_{\mu}$ uniquely as

$$
\gamma_{\mu}(\zeta)=\sigma_{\omega_{\nu}(0)}^{-1}(\zeta)=\frac{\left(1-\omega_{\nu}(0)\right) \zeta+\omega_{\nu}(0)\left(1-\overline{\omega_{\nu}(0)}\right)}{\left(1-\overline{\omega_{\nu}(0)}\right)+\zeta\left(1-\omega_{\nu}(0)\right) \overline{\omega_{\nu}(0)}}
$$

with $\sigma$ given by (2.3). Therefore, we have

$$
p \circ w_{\mu}(z)=\sigma_{w_{\nu}(0)} \circ w_{\nu} \circ p(z), \quad z \in \mathbb{U} \cup \mathbb{R} .
$$

Define $\mathfrak{P}: L^{\infty}\left(\mathbb{U}, \Gamma_{0}\right)_{1} \rightarrow S^{1} \backslash \operatorname{Homeo}_{q s}\left(S^{1}\right)$ to be $\mathfrak{K} \circ B$. Explicitly, it is given by

$$
\mathfrak{P}(\mu)=\left.\sigma_{w_{\nu}(0)} \circ w_{\nu}\right|_{S^{1}} .
$$

In other words, $\mathfrak{P}$ is defined by the commutative diagram

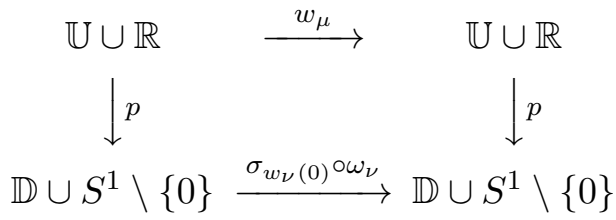

Lemma 3.1. If $\mu_{1}, \mu_{2} \in L^{\infty}\left(\mathbb{U}, \Gamma_{0}\right)_{1}$ and $\mu_{1} \sim \mu_{2}$, then $\mathfrak{P}\left(\mu_{1}\right)=\mathfrak{P}\left(\mu_{2}\right)$. 
Proof. If $\mu_{1} \sim \mu_{2}$, then $\left.w_{\mu_{1}}\right|_{\mathbb{R}}=\left.w_{\mu_{2}}\right|_{\mathbb{R}}$. From (3.4), it is easily deduced that then $\left.\sigma_{w_{\nu_{1}}(0)} \circ w_{\nu_{1}}\right|_{S^{1}}=\left.\sigma_{w_{\nu_{2}}(0)} \circ w_{\nu_{2}}\right|_{S^{1}}$, i.e., $\mathfrak{P}\left(\mu_{1}\right)=\mathfrak{P}\left(\mu_{2}\right)$.

From this lemma, we find that the map $\mathfrak{P}$ descends to the map $\mathcal{B}$ : $L^{\infty}\left(\mathbb{U}, \Gamma_{0}\right)_{1} / \sim=T\left(\Gamma_{0}\right) \longrightarrow S^{1} \backslash \operatorname{Homeo}_{q s}\left(S^{1}\right)$. Finally, the Bers isomorphism $\mathfrak{B}: T\left(\Gamma_{0}\right) \rightarrow F(1)$ is given by $\mathfrak{B}=\mathfrak{T}^{-1} \circ \mathcal{B}$. Working out explicitly,

$$
T\left(\Gamma_{0}\right) \ni[\mu] \stackrel{\mathfrak{B}}{\longrightarrow}\left([\nu], f^{\nu}(0)\right) \in F(1) .
$$

From (3.1) and the holomorphic dependance of $f^{\nu}(0)$ on $\nu$, we conclude immediately that $\mathfrak{B}$ is a holomorphic mapping.

On the other hand, by Remark 2.1] definition (2.1), the identification $F(1) \simeq S^{1} \backslash \operatorname{Homeo}_{q s}\left(S^{1}\right)$ and the commutative diagram (3.5) restricted to maps between $\mathbb{R}$ and $S^{1}$, we see that Bers isomorphism is nothing but a correspondence between quasi-symmetric homeomorphisms on the unit circle that fix the point 1 and the lifting of these homeomorphisms to the universal cover of $S^{1}$, i.e. $\mathbb{R}$, under the covering map $p: \mathbb{R} \rightarrow S^{1}$. It immediately follows that

Lemma 3.2. The mapping $\mathcal{B}: T\left(\Gamma_{0}\right) \rightarrow S^{1} \backslash$ Homeo $_{q s}\left(S^{1}\right)$ is a bijection.

Moreover, from the commutative diagram

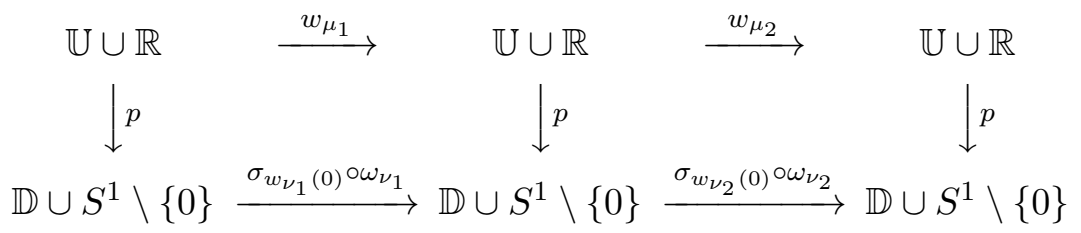

we conclude immediately that

Lemma 3.3. The mapping $\mathcal{B}: T\left(\Gamma_{0}\right) \rightarrow S^{1} \backslash$ Homeo $_{q s}\left(S^{1}\right)$ is a group homomorphism.

Grouping together, we have shown that

Theorem 3.4. The Bers isomorphism $\mathfrak{B}: T\left(\Gamma_{0}\right) \rightarrow F(1)$ is a biholomorphism between complex manifolds and a group isomorphism.

\section{The DeRIVATIVE MAPPING}

In this section, we study the derivative of the Bers isomorphism at the origin. The following theorem gives a significance relation between the Fourier coefficients of cusp forms and Fourier coefficients of vector fields on $S^{1}$.

Theorem 4.1. The derivative of the mapping $\mathfrak{P}: T\left(\Gamma_{0}\right) \rightarrow H_{o m e o}\left(S^{1}\right) / S^{1}$ at the origin is given by the following linear isomorphism

$$
\begin{aligned}
D_{0} \mathfrak{P}: \Omega^{-1,1}\left(\mathbb{U}, \Gamma_{0}\right) & \longrightarrow T_{0}\left(S^{1} \backslash \text { Homeo }_{q s}\left(S^{1}\right)\right) \\
-2 y^{2} \sum_{n=1}^{\infty} \overline{\alpha_{n} \exp (2 \pi i n z)} & \mapsto \frac{i}{4 \pi^{2}}\left(\sum_{n=1}^{\infty} \frac{\alpha_{n}}{n^{3}} e^{i n \theta}-\sum_{n=1}^{\infty} \frac{\bar{\alpha}_{n}}{n^{3}} e^{-i n \theta}\right)+c_{0} .
\end{aligned}
$$


where

$$
c_{0}=\frac{1}{4 \pi^{2} i}\left(\sum_{n=1}^{\infty} \frac{\alpha_{n}}{n^{3}}-\sum_{n=1}^{\infty} \frac{\bar{\alpha}_{n}}{n^{3}}\right) .
$$

Proof. Given $\mu \in \Omega^{-1,1}\left(\mathbb{U}, \Gamma_{0}\right)$, for $t$ in a neighborhood of 0 , we consider the one parameter family $w_{t \mu}$. Let $v_{t \mu}=\sigma_{\omega_{t \nu}(0)} \circ \omega_{t \nu}$, where $\mu$ and $\nu$ are related by (3.1) and by definition $\mathfrak{P}([t \mu])=\left.v_{t \mu}\right|_{S^{1}}$. From (3.5), we have

$$
v_{t \mu} \circ p(z)=p \circ w_{t \mu}(z), \quad z \in \mathbb{U} \cup \mathbb{R} .
$$

It is well known that (see e.g. Ahl87, Nag88) $w_{t \mu}$ is real analytic in $\mathbb{U}$ and therefore $v_{t \mu}$ is real analytic in $\mathbb{D}$. Taking derivative with respect to $t$ and putting $t=0$, we have

$$
\dot{v}_{\mu} \circ p(z)=p^{\prime}(z) \dot{w}_{\mu}(z), \quad z \in \mathbb{U}
$$

where $\dot{w}_{\mu}=\left.\frac{d}{d t}\right|_{t=0} w_{t \mu}$ and $\dot{v}_{\mu}=\left.\frac{d}{d t}\right|_{t=0} v_{t \mu}$. By continuity, (4.1) still holds on $\mathbb{R}$. Let

$$
\Phi_{\mu}(z)=\frac{i}{8 \pi^{3}} \sum_{n=1}^{\infty} \frac{\alpha_{n}}{n^{3}} \exp (2 \pi i n z)
$$

so that $\mu(z)=-2 y^{2} \overline{\Phi_{\mu}^{\prime \prime \prime}(z)}$. By a well-known theorem of Ahlfors [Ahl61, we have

$$
\dot{w}_{\mu}(z)=\frac{(z-\bar{z})^{2}}{2} \overline{\Phi_{\mu}^{\prime \prime}(z)}+(z-\bar{z}) \overline{\Phi_{\mu}^{\prime}(z)}+\overline{\Phi_{\mu}(z)}+\Phi_{\mu}(z)+p(z)+\overline{p(z)},
$$

where $p(z)$ is a polynomial of degree two. Since $w_{t \mu}$ fixes $0,1, \infty$, we have $\dot{w}_{\mu}$ vanishes at $0,1, \infty$, and we find that $p(z)$ is a constant and is equal to $p(z)=p(0)=-\Phi(0)$. Let

$$
\sum_{n \in \mathbb{Z}} c_{n} e^{i n \theta}=D_{0} \mathfrak{P}(\mu)=\left.\left(\frac{\dot{v}_{\mu}}{i z}\right)\right|_{S^{1}} .
$$

Restricted to $\mathbb{R}$, equation (4.1) and (4.2) give us

$i \exp (2 \pi i x) \sum_{n \in \mathbb{Z}} c_{n} \exp (2 \pi i n x)=2 \pi i \exp (2 \pi i x)\left(\overline{\Phi_{\mu}(x)}+\Phi_{\mu}(x)+p(x)+\overline{p(x)}\right)$.

Therefore,

$\sum_{n \in \mathbb{Z}} c_{n} \exp (2 \pi i n x)=\frac{i}{4 \pi^{2}} \sum_{n=1}^{\infty} \frac{\alpha_{n}}{n^{3}} \exp (2 \pi i n x)-\frac{i}{4 \pi^{2}} \sum_{n=1}^{\infty} \frac{\bar{\alpha}_{n}}{n^{3}} \exp (-2 \pi i n x)+c_{0}$,

where

$$
c_{0}=-2 \pi(\Phi(0)+\overline{\Phi(0)}) .
$$

This implies the assertion of the theorem. 
Remark 4.2. In [Teo04], we proved that if $v=\sum_{n \in \mathbb{Z}} c_{n} e^{i n \theta} \in T_{0}\left(S^{1} \backslash \operatorname{Homeo}_{q s}\left(S^{1}\right)\right)$, then

$$
\sum_{n=1}^{\infty} n^{\alpha}\left|c_{n}\right|^{2}<\infty
$$

for all $\alpha<2$. Theorem 4.1 then implies the series $\sum_{n=1}^{\infty}\left|\alpha_{n}\right|^{2} n^{-s}$ converges absolutely for all $\operatorname{Re} s>4$, which is a well-known result if $\phi=$ $\sum_{n=1}^{\infty} \alpha_{n} \exp (2 \pi i n z)$ is a cusp form of a cofinite Fuchsian group.

Theorem 4.3. The derivative of the Bers isomorphism $\mathfrak{B}: T\left(\Gamma_{0}\right) \rightarrow F(1)$ at the origin is given by the following linear isomorphism

$$
\begin{aligned}
D_{0} \mathfrak{B}: \Omega^{-1,1}\left(\mathbb{U}, \Gamma_{0}\right) & \longrightarrow \Omega^{-1,1}(\mathbb{D}) \oplus \mathbb{C} \\
-2 y^{2} \sum_{n=1}^{\infty} \overline{\alpha_{n} \exp (2 \pi i n z)} & \mapsto\left(\frac{\left(1-|z|^{2}\right)^{2}}{8 \pi^{2}} \sum_{n=2}^{\infty} \frac{n^{3}-n}{n^{3}} \bar{\alpha}_{n} \bar{z}^{n-2},-\frac{\bar{\alpha}_{1}}{4 \pi^{2}}\right)
\end{aligned}
$$

Proof. Given $\mu \in \Omega^{-1,1}\left(\mathbb{U}, \Gamma_{0}\right)$, by definition, $D_{0} \mathfrak{B}(\mu)=(\lambda, a)$, where $\lambda$ is the projection of $\nu$ defined by (3.1) to harmonic Beltrami differentials, and $a=\dot{f}^{\nu}(0)=\left.\frac{d}{d t}\right|_{t=0} f^{t \nu}(0)$. We can compute $\lambda$ by using the formula of projection. Instead, we reason as follows. Let $\omega_{t \lambda}$ be the corresponding family of quasi-conformal mappings defined by $t \lambda$, for $t$ in a small neighborhood of 0 . Then by definition, $\left.\dot{\omega}_{\lambda}\right|_{S^{1}}=\left.\dot{\omega}_{\nu}\right|_{S^{1}}$, where as usual, $\dot{\omega}_{\lambda}=\left.\frac{d}{d t}\right|_{t=0} \omega_{t \lambda}$ and $\dot{\omega}_{\nu}=\left.\frac{d}{d t}\right|_{t=0} \omega_{t \nu}$. By definition, $\lambda=-\frac{\left(1-|z|^{2}\right)^{2}}{2} \overline{\phi_{\lambda}}$ for some $\phi_{\lambda} \in A_{\infty}(\mathbb{D})$. Let

$$
\Phi_{\lambda}(z)=\sum_{n=2}^{\infty} \beta_{n} z^{n+1}, \quad \phi_{\lambda}(z)=\sum_{n=2}^{\infty}\left(n^{3}-n\right) \beta_{n} z^{n-2}
$$

so that $\Phi_{\lambda}^{\prime \prime \prime}=\phi_{\lambda}$. By the same formula of Ahlfors (4.2) applied to the disc model, we have

$$
\dot{\omega}_{\lambda}(z)=-\frac{\left(1-|z|^{2}\right)^{2}}{2} \overline{\Phi_{\lambda}^{\prime \prime}(z)}-z\left(1-|z|^{2}\right) \overline{\Phi_{\lambda}^{\prime}(z)}-z^{2} \overline{\Phi_{\lambda}(z)}+\Phi_{\lambda}(z)+q(z)
$$

where $q(z)=a_{0}+a_{1} z+a_{2} z^{2}, a_{0}=-\bar{a}_{2}$ and $a_{1}$ is purely imaginary. Restricted to $S^{1}$, we have

$$
\dot{\omega}_{\nu}(z)=\dot{\omega}_{\lambda}(z)=-\sum_{n=2}^{\infty} \bar{\beta}_{n} z^{1-n}+\sum_{n=2}^{\infty} \beta_{n} z^{n+1}+a_{0}+a_{1} z+a_{2} z^{2} .
$$

On the other hand, from the relation $\omega_{t \nu}=g_{t \nu}^{-1} \circ f^{t \nu}$, we have

$$
\dot{\omega}_{\nu}(z)=-\dot{g}_{\nu}(z)+\dot{f}^{\nu}(z), \quad z \in \mathbb{C} .
$$

From the fact that $g_{t \nu}(0)=0$ and the form of $f^{t \nu}$ given by (2.2), we find that $a_{0}=0$ and hence $a_{2}=0$. Equation (4.5) also gives us $\dot{f}^{\nu}(0)=\dot{w}_{\nu}(0)$. 
Now using the definition $v_{t \mu}=\sigma_{\omega_{t \nu}(0)} \circ \omega_{t \nu}$ and (2.3), we have

$$
\begin{aligned}
\dot{v}_{\mu}(z) & =\left(\left.\frac{d}{d t}\right|_{t=0} \sigma_{\omega_{t \nu}(0)}\right)(z)+\dot{\omega}_{\nu}(z) \\
& =-\dot{\omega}_{\nu}(0)+\left(\dot{\omega}_{\nu}(0)-\overline{\dot{\omega}_{\nu}(0)}\right) z+\overline{\dot{\omega}_{\nu}(0)} z^{2}+\dot{\omega}_{\nu}(z) .
\end{aligned}
$$

Comparing this equation with equations (4.3), (4.4) and the result of Theorem 4.1. we find that

$$
\dot{w}_{\nu}(0)=-i \bar{c}_{1}=-\frac{1}{4 \pi^{2}} \bar{\alpha}_{1} \quad \text { and for } n \geq 2, \quad \beta_{n}=i c_{n}=-\frac{1}{4 \pi^{2}} \frac{\alpha_{n}}{n^{3}} .
$$

The assertion of the theorem follows.

Remark 4.4. $T\left(\Gamma_{0}\right)$ can be thought of as the parameter space of all the onepunctured surfaces. This theorem implies that the tangent vector on $T\left(\Gamma_{0}\right)$ that purely 'moves the puncture' corresponds to the automorphic form which has only the first coefficient nonzero. On the other hand, the automorphic form with vanishing first coefficient does not 'move the puncture'.

\section{TAKhtajan-Zograf metric AND Velling-Kirillov metriC}

In Teo04 and TT03, we have studied some properties of the VellingKirillov metric on the universal Teichmüller curve $F(1)$. We can pull back this metric to $T\left(\Gamma_{0}\right)$ via the Bers isomorphism, which we still call the VellingKirillov metric. A straight-forward computation gives

Theorem 5.1. On $T\left(\Gamma_{0}\right)$, the Velling-Kirillov metric is a multiple of the Takhtajan-Zograf metric. More precisely, for $\mu_{1}, \mu_{2} \in \Omega^{-1,1}\left(\mathbb{U}, \Gamma_{0}\right)$,

$$
\left\langle\mu_{1}, \mu_{2}\right\rangle_{V K}=\frac{2 \pi}{3} \iint_{\Gamma_{0} \backslash \mathbb{U}} \mu_{1}(z) \overline{\mu_{2}(z)} d^{2} z=\frac{2 \pi}{3}\left\langle\mu_{1}, \mu_{2}\right\rangle_{T Z} .
$$

Proof. By definition and Theorem 4.1

$$
\|\mu\|_{V K}^{2}=\sum_{n=1}^{\infty} n\left|\frac{i}{4 \pi^{2}} \frac{\alpha_{n}}{n^{3}}\right|^{2}=\frac{1}{16 \pi^{4}} \sum_{n=1}^{\infty} \frac{\left|\alpha_{n}\right|^{2}}{n^{5}} .
$$

Compare to the definition (2.4) of the Takhtajan-Zograf metric and (2.5), the result follows.

Since we have proved in Teo04 that the Velling-Kirillov metric is Kähler, we obtain immediately

Corollary 5.2. The Takhtajan-Zograf metric on $T\left(\Gamma_{0}\right)$ is a right-invariant Kähler metric.

Remark 5.3. Let $G$ and $\dot{G}$ be Fuchsian groups as in Theorem 1.1 Although $T(\dot{G})$ and $F(G)$ are submanifolds of $T\left(\Gamma_{0}\right)$ and $F(1)$ respectively, the Bers isomorphism $\mathfrak{B}: T\left(\Gamma_{0}\right) \rightarrow F(1)$ we study in Section 3 does not induce the Bers isomorphism $\mathfrak{B}_{G}: T(\dot{G}) \rightarrow F(G)$. In fact, when $G \neq\{$ id $\}$, we do not have an explicit expression of the biholomorphism $h: \mathbb{U} / \dot{G} \rightarrow(\mathbb{U} / G) \backslash\{\hat{a}\}$ of 
Theorem 1.1 and thus we cannot write down the isomorphism $\mathfrak{B}_{G}: T(\dot{G}) \rightarrow$ $F(G)$ and its derivative mapping explicitly as in Section 4 . Nevertheless, it will be interesting to compare the Velling-Kirillov metric on $F(G)$ to the Takhtajan-Zograf metric on $T(\dot{G})$ under Bers isomorphism $\mathfrak{B}_{G}$.

Bowick and Rajeev BR87 and Kirillov and Yuriev [KY87 have calculated the curvature tensor of the Velling-Kirillov metric on the homogenous space $S^{1} \backslash \operatorname{Diff}_{+}\left(S^{1}\right)$, a natural submanifold of $S^{1} \backslash \operatorname{Homeo}_{q s}\left(S^{1}\right) \simeq F(1)$. Via the Bers isomorphism $\mathfrak{B}: T\left(\Gamma_{0}\right) \rightarrow F(1)$, we can use their result to find the curvature tensor of the Takhtajan-Zograf metric on $T\left(\Gamma_{0}\right)$ and study the properties of the curvature. This will in turn give the curvature properties of the Takhtajan-Zograf metric on the Teichmüller spaces of cofinite punctured Riemann surfaces. We will consider this question in a subsequent paper.

Acknowledgments. I would like to thank Leon Takhtajan for the valuable discussions when this work was done. This work was partially supported by MMU internal funding PR/2006/0590.

\section{REFERENCES}

[Ahl61] Lars V. Ahlfors, Some remarks on Teichmüller's space of Riemann surfaces, Ann. of Math. (2) $\mathbf{7 4}$ (1961), 171-191.

[Ahl87] _ Lectures on quasiconformal mappings, Wadsworth \& Brooks/Cole Advanced Books \& Software, Monterey, CA, 1987, With the assistance of Clifford J. Earle, Jr., Reprint of the 1966 original.

[Ber73] Lipman Bers, Fiber spaces over Teichmüller spaces, Acta. Math. 130 (1973), $89-126$.

[BR87] M. J. Bowick and S. G. Rajeev, String theory as the Kähler geometry of loop space, Phys. Rev. Lett. 58 (1987), no. 6, 535-538.

[KY87] A. A. Kirillov and D. V. Yur'ev, Kähler geometry of the infinite-dimensional homogeneous space $M=\operatorname{diff}_{+}\left(S^{1}\right) / \operatorname{rot}\left(S^{1}\right)$, Funktsional. Anal. i Prilozhen. 21 (1987), no. 4, 35-46, 96.

[Leh87] Olli Lehto, Univalent functions and Teichmüller spaces, Springer-Verlag, New York, 1987.

[Nag88] Subhashis Nag, The complex analytic theory of Teichmüller spaces, John Wiley \& Sons Inc., New York, 1988, A Wiley-Interscience Publication.

[Teo04] Lee-Peng Teo, Velling-Kirillov metric on the universal Teichmüller curve, Journal d' Analyse Mathematique 93 (2004), 271-308.

[TT03] Leon A. Takhtajan and Lee-Peng Teo, Weil-Petersson metric on the universal Teichmüller space I: Curvature properties and Chern forms, Preprint arXiv: math.CV/0312172 (2003).

[TZ91] L. A. Takhtajan and P. G. Zograf, A local index theorem for families of $\bar{\partial}$ operators on punctured Riemann surfaces and a new Kähler metric on their moduli spaces, Comm. Math. Phys. 137 (1991), no. 2, 399-426.

Faculty of Information Technology, Multimedia Univerity, Jalan Multimedia, 63100, Cyberjaya, Selangor, Malaysia

E-mail address: lpteo@mmu.edu.my 